\title{
A Decomposable Branching Process in a Markovian Environment
}

\author{
Vladimir Vatutin, ${ }^{1}$ Elena Dyakonova, ${ }^{1}$ Peter Jagers, $^{2}$ \\ and Serik Sagitov ${ }^{2}$ \\ ${ }^{1}$ Steklov Mathematical Institute, Russian Academy of Sciences, Moscow 119991, Russia \\ ${ }^{2}$ Chalmers University of Technology and University of Gothenberg, 41296 Gothenburg, Sweden
}

Correspondence should be addressed to Serik Sagitov, serik@chalmers.se

Received 28 September 2012; Accepted 18 December 2012

Academic Editor: Peter Olofsson

Copyright (C) 2012 Vladimir Vatutin et al. This is an open access article distributed under the Creative Commons Attribution License, which permits unrestricted use, distribution, and reproduction in any medium, provided the original work is properly cited.

A population has two types of individuals, with each occupying an island. One of those, where individuals of type 1 live, offers a variable environment. Type 2 individuals dwell on the other island, in a constant environment. Only one-way migration $(1 \rightarrow 2)$ is possible. We study then asymptotics of the survival probability in critical and subcritical cases.

\section{Introduction}

Multitype branching process in random environment is a challenging topic with many motivations from population dynamics (see, e.g., [1-3]). Very little is known in the general case and in this paper we consider a particular two-type branching process with two key restrictions: the process is decomposable and the final type individuals live in a constant environment.

The subject can be viewed as a stochastic model for the sizes of a geographically structured population occupying two islands. Time is assumed discrete, so that one unit of time represents a generation of individuals, some living on island 1 and others on island 2. Those on island 1 give birth under influence of a randomly changing environment. They may migrate to island 2 immediately after birth, with a probability again depending upon the current environmental state. Individuals on island 2 do not migrate and their reproduction law is not influenced by any changing environment. Our main concern is the survival probability of the whole population.

An alternative interpretation of the model under study might be a population (type 1) subject to a changing environment, say in the form of a predator population of stationary 
but variable size. Its individuals may mutate into a second type, no longer exposed to the environmental variation (the predators do not regard the mutants as prey). Our framework may be also suitable for modeling early carcinogenesis, a process in which mutant clones repeatedly arise and disappear before one of them becomes established [4,5]. See [6] for yet another possible application.

The model framework is that furnished by Bienaymé-Galton-Watson (BGW) processes with individuals living one unit of time and replaced by random numbers of offspring which are conditionally independent given the current state of the environment. We refer to such individuals as particles in order to emphasize the simplicity of their lives. Particles of type 1 and 2 are distinguished according to the island number they are occupying at the moment of observation. Our main assumptions are

(i) particles of type 1 form a critical or subcritical branching process in a random environment,

(ii) particles of type 2 form a critical branching process which is independent of the environment.

Let $X_{n}$ and $Z_{n}$ be the numbers of particles of type 1 and of type 2, respectively, present at time $n$. Throughout this paper it is assumed (unless otherwise specified) that $X_{0}=1$ and $Z_{0}=0$. We investigate asymptotics of the survival probability $\mathbb{P}\left[X_{n}+Z_{n}>0\right]$ as $n \rightarrow \infty$. In all cases addressed here we have

$$
\mathbb{P}\left[X_{n}>0\right]=o\left(\mathbb{P}\left[X_{n}+Z_{n}>0\right]\right) .
$$

Therefore, in view of

$$
\mathbb{P}\left[Z_{n}>0\right] \leq \mathbb{P}\left[X_{n}+Z_{n}>0\right] \leq \mathbb{P}\left[X_{n}>0\right]+\mathbb{P}\left[Z_{n}>0\right],
$$

we focus on the asymptotic behavior of $\mathbb{P}\left[Z_{n}>0\right]$.

In Section 2 we recall known facts for constant environments. They will then be compared to the results of this paper on random environments. In Section 3.1 we describe IID environments (Independent and Identically Distributed environmental states) and then in Section 3.2 Markovian environments. The main results of the paper are

(i) Theorem 4.1 in Section 4 on the critical case with an IID environment,

(ii) Theorem 5.1 in Section 5 on the subcritical case with an IID environment,

(iii) Theorem 6.1 in Section 6 on the critical case with a Markovian environment,

(iv) Theorem 7.1 in Section 7 on subcritical case with a Markovian environment.

Theorems 6.1 and 7.1 treating the case of Markovian environment are extensions of Theorems 4.1 and 5.1 obtained under rather restrictive conditions and yielding qualitatively the same asymptotic behavior as in the case of IID environment.

Notation 1. In asymptotic formulae constants denoted by the same letter $c$ are always assumed to be fixed and independent of the parameter that tends to infinity (or zero). 


\section{Two-Type Decomposable Branching Processes}

Consider a two-type BGW process initiated at time zero by a single individual of type 1 . We focus on the decomposable case where type 1 particles may produce particles of types 1 and 2 , while type 2 particles can give birth only to type 2 particles. Put

(i) $Y_{n}:=$ the number of type 2 daughters produced by the particles of type 1 present at time $n$, in particular, $Y_{0}=Z_{1}$,

(ii) $T:=$ the first time $n$ when $X_{n}=0$, so that $\{T>n\}=\left\{X_{n}>0\right\}$,

(iii) $S_{n}:=\sum_{k=0}^{n-1} X_{k}$, so that $S_{\mathrm{T}}$ gives the total number ever of type 1 particles,

(iv) $W_{n}:=\sum_{k=0}^{n-1} Y_{k}$, so that $W_{\mathrm{T}}$ gives the total number of type 2 daughters produced by all $S_{\mathrm{T}}$ particles of type 1 .

The aim of this section is to summarize what is already known about such branching processes in the case of a constant environment. This will pave our way in terms of notation and basic manipulation with generating functions towards branching processes in IID random and then Markovian environments.

If the environment is constant from generation to generation, two-type decomposable BGW processes are fully described by a pair of probability generating functions

$$
\begin{gathered}
f\left(s_{1}, s_{2}\right):=\mathbb{E}\left[s_{1}^{\xi_{1}} s_{2}^{\xi_{2}}\right], \\
h(s):=\mathbb{E}\left[s^{\eta}\right],
\end{gathered}
$$

where $\xi_{1}$ and $\xi_{2}$ represent the numbers of daughters of type 1 and 2 of a mother of type 1 , while $\eta$ stands for the number of daughters (necessarily of type 2 ) of a mother of type 2 . Let

$$
\begin{aligned}
& \mu_{1}:=\mathbb{E}\left[\xi_{1}\right]=\left.\frac{\partial f\left(s_{1}, s_{2}\right)}{\partial s_{1}}\right|_{s_{1}=s_{2}=1}, \\
& \mu_{2}:=\mathbb{E}\left[\xi_{1}\left(\xi_{1}-1\right)\right]=\left.\frac{\partial^{2} f\left(s_{1}, s_{2}\right)}{\partial s_{1}^{2}}\right|_{s_{1}=s_{2}=1}, \\
& \theta_{1}:=\mathbb{E}\left[\xi_{2}\right]=\left.\frac{\partial f\left(s_{1}, s_{2}\right)}{\partial s_{2}}\right|_{s_{1}=s_{2}=1}, \\
& \theta_{2}:=\mathbb{E}\left[\xi_{2}\left(\xi_{2}-1\right)\right]=\left.\frac{\partial^{2} f\left(s_{1}, s_{2}\right)}{\partial s_{2}^{2}}\right|_{s_{1}=s_{2}=1}, \\
& m_{1}:=\mathbb{E}[\eta]=h^{\prime}(1), \\
& m_{2}:=\mathbb{E}[\eta(\eta-1)]=h^{\prime \prime}(1)
\end{aligned}
$$

be the first two moments of the reproduction laws. Concerning the second type of particles we assume that

$$
m_{1}=1, \quad m_{2} \in(0, \infty),
$$


implying that the probability of extinction

$$
Q_{n}:=\mathbb{P}\left[Z_{n}=0 \mid X_{0}=0, Z_{0}=1\right]
$$

(of a single-type BGW process evolving in constant environment with the probability generating function $h(s))$ satisfies [7, Chapter I.9]

$$
1-Q_{n} \sim \frac{2}{m_{2} n}, \quad n \longrightarrow \infty .
$$

It follows that

$$
a_{n}:=-\log f\left(1, Q_{n}\right) \sim 1-f\left(1, Q_{n}\right) \sim \frac{2 \theta_{1}}{m_{2} n}, \quad n \longrightarrow \infty
$$

We will be interested in two kinds of reproduction regimes for particles of type 1 , critical and subcritical. In the constant environment setting with $\mu_{2} \in(0, \infty)$, the critical case corresponds to $\mu_{1}=1$, and the subcritical case is given by $\mu_{1} \in(0,1)$. In the critical case with a constant environment we have

$$
\mathbb{P}\left[X_{n}>0\right]=\mathbb{P}[T>n] \sim \frac{2}{\mu_{2} n}, \quad n \longrightarrow \infty,
$$

and according to $[8$, Theorem 1$]$

$$
\mathbb{P}\left[X_{n}+Z_{n}>0\right] \sim \mathbb{P}\left[Z_{n}>0\right] \sim \frac{2 \sqrt{\theta_{1}}}{\sqrt{m_{2} \mu_{2} n}}, \quad n \longrightarrow \infty .
$$

Next we outline a proof of (2.8) based on the representation

$$
\begin{aligned}
\mathbb{P}\left[Z_{n}>0\right] & =\mathbb{E}\left[1-\prod_{k=0}^{n-1} Q_{n-k}^{Y_{k}}\right]=\mathbb{E}\left[1-\prod_{k=0}^{n-1} f^{X_{k}}\left(1, Q_{n-k}\right)\right] \\
& =\mathbb{E}\left[1-e^{-\sum_{k=0}^{n-1} X_{k} a_{n-k}}\right]
\end{aligned}
$$

preparing for the proof in the random environment case, to be given in Section 4 . Thanks to (2.7) and (1.2), it is enough to verify that

$$
\mathbb{P}\left[Z_{n}>0\right] \sim \frac{2 \sqrt{\theta_{1}}}{\sqrt{m_{2} \mu_{2} n}}, \quad n \longrightarrow \infty,
$$

in order to prove (2.8). However, by the branching property the total progeny of a single-type branching process $S_{\mathrm{T}}$ is 1 plus $\xi_{1}$ independent daughter copies of $S_{\mathrm{T}}$. In terms of the Laplace transform

$$
\phi(\lambda)=e^{-\lambda} f(\phi(\lambda), 1)
$$


where $\phi(\lambda):=\mathbb{E}\left[e^{-\lambda S_{\mathrm{T}}}\right]$. As $\lambda \rightarrow 0$, a Taylor expansion of $f(\phi(\lambda), 1)$ as a function of $1-\phi(\lambda)$ yields

$$
1-\phi(\lambda)=1-e^{-\lambda}+e^{-\lambda} \mu_{1}(1-\phi(\lambda))-e^{-\lambda} \frac{\mu_{2}}{2}(1-\phi(\lambda))^{2}(1+o(1))
$$

For $\mu_{1}=1$, after removing the negligible terms, we get a quadratic equation whose solution shows that

$$
1-\phi(\lambda) \sim \sqrt{\frac{2 \lambda}{\mu_{2}}}, \quad \lambda \longrightarrow 0
$$

Replacing $\lambda$ by $a_{n}$ and using (2.6), we obtain

$$
\mathbb{E}\left[1-e^{-S_{\mathrm{T}} a_{n}}\right] \sim \frac{2 \sqrt{\theta_{1}}}{\sqrt{m_{2} \mu_{2} n}}, \quad n \longrightarrow \infty
$$

It remains to verify, see (2.9), that

$$
\mathbb{E}\left[1-e^{-\sum_{k=0}^{n-1} X_{k} a_{n-k}}\right] \sim \mathbb{E}\left[1-e^{-S_{\mathrm{T}} a_{n}}\right], \quad n \longrightarrow \infty
$$

This holds, indeed, since by (2.7) and for any fixed $\epsilon>0$ the probability $\mathbb{P}[T>n \epsilon]$ is much smaller than the target value of order $c / \sqrt{n}$. (In $[9,10]$ infinite second moments in decomposable two-type critical processes were allowed.)

On the other hand, in the subcritical case (2.12) implies that

$$
1-\phi(\lambda) \sim \frac{\lambda}{\left(1-\mu_{1}\right)}, \quad \lambda \longrightarrow 0
$$

so that by (2.6)

$$
\mathbb{E}\left[1-e^{-S_{\mathrm{T}} a_{n}}\right] \sim \frac{2 \theta_{1}}{m_{2}\left(1-\mu_{1}\right) n}, \quad n \longrightarrow \infty
$$

In view of $\mathbb{P}\left[X_{n}>0\right] \sim c \mu_{1}^{n}$ we conclude that in the subcritical case

$$
\mathbb{P}\left[X_{n}+Z_{n}>0\right] \sim \mathbb{P}\left[Z_{n}>0\right] \sim \frac{2 \theta_{1}}{m_{2}\left(1-\mu_{1}\right) n}, \quad n \longrightarrow \infty
$$

See [11] for a comprehensive study of subcritical decomposable branching processes in a constant environment. 


\section{Branching Processes in a Random Environment}

A randomly changing environment for BGW processes is modeled by a random sequence of probability generating functions for the offspring distributions of consecutive generations. Throughout this paper we assume that the offspring distribution for type 2 particles is the same across the different states of the environment and characterized by the same generating function $h(s)$. This restriction that greatly simplifies analysis still allows new interesting asymptotic regimes.

We consider two types of stationarily changing environments: IID and Markovian.

\subsection{IID Environment}

Our description of the IID environment case starts with a simple illustration based on just two alternative bivariate generating functions $f^{(1)}\left(s_{1}, s_{2}\right)$ and $f^{(2)}\left(s_{1}, s_{2}\right)$ with mean offspring numbers $\left(\mu_{1}^{(1)}, \theta_{1}^{(1)}\right)$ and $\left(\mu_{1}^{(2)}, \theta_{1}^{(2)}\right)$, respectively. We assume that at each time $n$ the environment is say "good" with probability $\pi_{1}$, so that type 1 particles reproduce independently according to the $f^{(1)}\left(s_{1}, s_{2}\right)$, and with probability $\pi_{2}=1-\pi_{1}$ the environment is "bad" and particles of type 1 reproduce according to the $f^{(2)}\left(s_{1}, s_{2}\right)$ law. In other words, the generating function $f\left(s_{1}, s_{2}\right)$ should be treated as a random function having distribution

$$
\mathbb{P}\left[f\left(s_{1}, s_{2}\right)=f^{(1)}\left(s_{1}, s_{2}\right)\right]=\pi_{1}, \quad \mathbb{P}\left[f\left(s_{1}, s_{2}\right)=f^{(2)}\left(s_{1}, s_{2}\right)\right]=\pi_{2} .
$$

In particular, the vector of the mean offspring numbers $\left(\mu_{1}, \theta_{1}\right)$ takes values $\left(\mu_{1}^{(1)}, \theta_{1}^{(1)}\right)$ and $\left(\mu_{1}^{(2)}, \theta_{1}^{(2)}\right)$ with probabilities $\pi_{1}$ and $\pi_{2}$.

More generally, our two-type branching process in an IID random environment is characterized (besides the fixed reproduction law $h(s)$ for the type 2 particles) by a sequence of generating functions $\left\{f_{n}\left(s_{1}, s_{2}\right)\right\}_{n=0}^{\infty}$ independently drawn from a certain distribution over probability generating functions so that

$$
f_{n}\left(s_{1}, s_{2}\right) \stackrel{d}{=} f\left(s_{1}, s_{2}\right)
$$

In this setting the respective conditional moments $\mu_{1}, \mu_{2}, \theta_{1}$, and $\theta_{2}$ should be treated as random variables. An important role is played by the random variable $\zeta:=\log \mu_{1}$ representing the step size of the so-called associated random walk [12] formed by the partial sums $\zeta_{0}+\cdots+$ $\zeta_{n-1}$ with $\zeta_{i} \stackrel{d}{=} \zeta$.

Notation 2. Characteristics of the reproduction law in generation $n$ are denoted by adding an extra lower index $n$ to the generic notation, like in (3.2).

\subsection{Markovian Environment}

One way to relax the IID assumption on the environment is to allow for Markovian dependence among its consecutive states. We implement this by modelling changes in terms of an irreducible aperiodic positive recurrent Markov chain $\left\{e_{n}\right\}_{n=0}^{\infty}$ with countably many states $\{1,2, \ldots\}$. Assuming a stationary initial distribution $\left(\pi_{1}, \pi_{2}, \ldots\right)$, we associate 
with each state $i$ of this chain a probability generating function $f^{(i)}\left(s_{1}, s_{2}\right)$, so that the changing environment for the branching process is governed by the sequence of identically reproduction laws

$$
f_{n}\left(s_{1}, s_{2}\right):=f^{\left(e_{n}\right)}\left(s_{1}, s_{2}\right), \quad n=0,1, \ldots
$$

with Markovian dependence. Due to the stationarity we can again write (3.2) and use the same notation for the marginal moments of the reproduction laws as in the IID case.

To build a bridge to the IID environment case we use an embedding through a sequence of regeneration moments $\left\{\tau_{k}\right\}_{k=0}^{\infty}$ defined as

$$
\tau_{0}:=0, \quad \tau_{k+1}:=\min \left\{n>\tau_{k}: e_{n}=e_{0}\right\}
$$

The times $\tau_{k+1}-\tau_{k}$ between consecutive regenerations are independent and all distributed as $\tau:=\tau_{1}$. The embedded process $\left(\widehat{X}_{n}, \widehat{Z}_{n}\right)$ defined as

$$
\left(\widehat{X}_{n}, \widehat{Z}_{n}\right):=\left(X_{\tau_{n}}, Z_{\tau_{n}}\right), \quad n=0,1, \ldots
$$

is a decomposable branching process in an IID environment with two types of particles $\hat{1}$ and $\widehat{2}$ and conditional reproduction generating functions

$$
\begin{aligned}
\widehat{f}\left(s_{1}, s_{2}\right) & :=f^{\left(e_{0}\right)}\left(f^{\left(e_{1}\right)}\left(\cdots\left(f^{\left(e_{\tau-1}\right)}\left(s_{1}, s_{2}\right), h\left(s_{2}\right)\right) \cdots\right), h_{\tau-1}\left(s_{2}\right)\right), \\
\widehat{h}(s) & :=h(h(\cdots h(s) \cdots))=h_{\tau}(s),
\end{aligned}
$$

where $h_{k}(s)$ stands for the $k$-fold iteration of $h(s)$.

Notation 3. For all characteristics of the embedded process $\left(\widehat{X}_{n}, \widehat{Z}_{n}\right)$ and related constants appearing in the asymptotic formulae we use the same notation as for the process $\left(X_{n}, Z_{n}\right)$ in the IID case just adding the hat sign.

The key difference from the IID case is that the reproduction law for the $\widehat{2}$-type particles is dependent on the random environment. However, this dependence is of specific nature which we are able to manage using the law of large numbers for renewal processes. Notice that on its own the $\widehat{2}$-type particles form a so-called degenerate critical branching process in an IID random environment [12]: its conditional offspring mean is deterministic $\widehat{m}_{1}=1$. Meanwhile, the conditional variance is random $\widehat{m}_{2}=\tau m_{2}$.

Taking the first- and second-order derivatives of (3.6), we can express the moments of the reproduction law of the embedded process in terms of the moments of the consecutive reproduction laws with Markovian dependence. In what follows we use Notation 2 again, 
while keeping in mind that the sequence $\left(\mu_{1, k}, \mu_{2, k}, \theta_{1, k}, \theta_{2, k}\right)_{k=0}^{\tau-1}$ now consists of dependent random vectors. It can be shown that

$$
\begin{gathered}
\widehat{\mu}_{1}=\prod_{k=0}^{\tau-1} \mu_{1, k}, \quad \widehat{\mu}_{2}=\widehat{\mu}_{1} \sum_{k=0}^{\tau-1} \frac{\mu_{2, k}}{\mu_{1, k}} \prod_{i=k+1}^{\tau-1} \mu_{1, i}, \\
\widehat{\theta}_{1}=\sum_{k=0}^{\tau-1} \theta_{1, k} \prod_{i=0}^{k-1} \mu_{1, i}
\end{gathered}
$$

where, as usual, the product of the elements of an empty set is one. Furthermore, setting

$$
A_{k, n}=\sum_{j=k}^{n} \theta_{1, j} \prod_{i=k}^{j-1} \mu_{1, i}
$$

we can write

$$
\widehat{\theta}_{2}=\sum_{k=0}^{\tau-1} \theta_{2, k} \prod_{i=0}^{k-1} \mu_{1, i}+\sum_{k=0}^{\tau-2}\left\{\mu_{2, k} A_{k+1, \tau-1}^{2}+2 \mu_{1, k} \theta_{1, k} A_{k+1, \tau-1}+\sigma^{2}(\tau-1-k) \theta_{1, k}\right\} \prod_{i=0}^{k-1} \mu_{1, i} .
$$

Lemma 3.1. Let

$$
\sum_{k=0}^{\infty} \mathbb{E}\left[\left|\zeta_{k}\right| 1_{\{\tau \geq k+1\}}\right]<\infty
$$

For the following sum of a random number of random variables

$$
\widehat{\zeta}:=\sum_{k=0}^{\tau-1} \zeta_{k}, \quad \zeta_{k}:=\log \mu_{1, k}=\zeta\left(e_{k}\right)
$$

a version of the Wald identity holds: $\mathbb{E}[\widehat{\zeta}]=\mathbb{E}[\tau] \mathbb{E}[\zeta]$.

Proof. For any state $j$ consider the function

$$
\mu_{j}(i):=\sum_{n=0}^{\infty} \mathbb{P}\left[e_{n}=i, \tau>n \mid e_{0}=j\right]
$$

According to [13, Theorem 6.5.2] this defines a stationary measure which is necessarily of the form $\mu_{j}(i)=c_{j} \pi_{i}$. The constants $c_{j}$ are such that

$$
\begin{aligned}
\sum_{j=1}^{\infty} \pi_{j} c_{j} & =\sum_{j=1}^{\infty} \pi_{j} \sum_{i=1}^{\infty} \mu_{j}(i)=\sum_{j=1}^{\infty} \pi_{j} \sum_{n=0}^{\infty} \sum_{i=1}^{\infty} \mathbb{P}\left[e_{n}=i, \tau>n \mid e_{0}=j\right] \\
& =\sum_{j=1}^{\infty} \pi_{j} \mathbb{E}\left[\tau \mid e_{0}=j\right]=\mathbb{E}[\tau] .
\end{aligned}
$$


It follows that

$$
\begin{aligned}
\mathbb{E}[\hat{\zeta}] & =\sum_{k=1}^{\infty} \sum_{n=0}^{k-1} \mathbb{E}\left[\zeta_{n} 1_{\{\tau=k\}}\right]=\sum_{n=0}^{\infty} \mathbb{E}\left[\zeta\left(e_{n}\right) 1_{\{\tau>n\}}\right] \\
& =\sum_{n=0}^{\infty} \sum_{i=1}^{\infty} \zeta(i) \mathbb{P}\left[e_{n}=i, \tau>n\right]=\sum_{i=1}^{\infty} \zeta(i) \sum_{j=1}^{\infty} \pi_{j} \mu_{j}(i) \\
& =\sum_{i=1}^{\infty} \zeta(i) \pi_{i} \mathbb{E}[\tau]=\mathbb{E}[\tau] \mathbb{E}[\zeta] .
\end{aligned}
$$

Developing the example of two environmental states from Section 3.1, let us consider a Markov chain $\left\{e_{n}\right\}_{n=0}^{\infty}$ with transition probabilities

$$
\left(\begin{array}{cc}
1-d \pi_{2} & d \pi_{2} \\
d \pi_{1} & 1-d \pi_{1}
\end{array}\right), \quad 0<d<\min \left(\frac{1}{\pi_{1}}, \frac{1}{\pi_{2}}\right)
$$

and a stationary distribution $\left(\pi_{1}, \pi_{2}\right)$. (Notice that $d=1$ corresponds to the IID case.) Under stationarity the regeneration time satisfies

$$
\begin{aligned}
\mathbb{P}[\tau=1] & =\pi_{1}\left(1-d \pi_{2}\right)+\pi_{2}\left(1-d \pi_{1}\right)=1-2 \pi_{1} \pi_{2} d, \\
\mathbb{P}[\tau=k] & =\pi_{1} d \pi_{2}\left(1-d \pi_{1}\right)^{k-2} d \pi_{1}+\pi_{2} d \pi_{1}\left(1-d \pi_{2}\right)^{k-2} d \pi_{2} \\
& =d \pi_{1} \pi_{2}\left(d \pi_{1}\left(1-d \pi_{1}\right)^{k-2}+d \pi_{2}\left(1-d \pi_{2}\right)^{k-2}\right), \quad k \geq 2,
\end{aligned}
$$

implying that

$$
\mathbb{E}[\tau-1]=1, \quad \mathbb{E}[\tau(\tau-1)]=\frac{2}{d \pi_{1} \pi_{2}}-\frac{4}{d}
$$

If $\left(b_{1}, b_{2}\right)$ are the two possible values for $\zeta$, we can write $\mathbb{E}[\zeta]=\pi_{1} b_{1}+\pi_{2} b_{2}$ and

$$
\begin{aligned}
\mathbb{E}[\hat{\zeta}]= & \mathbb{E}[\mathbb{E}[\hat{\zeta} \mid \tau] ; \tau=1]+\mathbb{E}[\mathbb{E}[\hat{\zeta} \mid \tau] ; \tau \geq 2] \\
= & \pi_{1}\left(1-d \pi_{2}\right) b_{1}+\pi_{2}\left(1-d \pi_{1}\right) b_{2} \\
& +\pi_{1} d \pi_{2}\left(b_{1}+\frac{b_{2}}{d \pi_{1}}\right)+\pi_{2} d \pi_{1}\left(b_{2}+\frac{b_{1}}{d \pi_{2}}\right)=2 \mathbb{E}[\zeta],
\end{aligned}
$$

in full agreement with Lemma 3.1. 


\section{Critical Processes in IID Environment}

The single-type critical branching process with an IID environment displays an asymptotic behavior that is in stark contrast with the constant environment formula (2.7). According to $[14$, Theorem 1], if

$$
\begin{gathered}
\mathbb{E}[\zeta]=0, \quad \operatorname{Var}[\zeta] \in(0, \infty), \\
\mathbb{E}\left[\mu_{2} \mu_{1}^{-2}\left(1+\max \left(0, \log \mu_{1}\right)\right)\right]<\infty,
\end{gathered}
$$

then for some positive constant $c$

$$
\mathbb{P}\left[X_{n}>0\right]=\mathbb{P}[T>n] \sim \frac{c}{\sqrt{n}}, \quad n \longrightarrow \infty
$$

(A much more general limit theorem is obtained in [12].) The following theorem shows that in the decomposable case the difference between constant and random environments is even more striking. For constant environments the survival probability decays as $c / \sqrt{n}$, see (2.8), but in random environments the decay is like $c / \log n$.

Theorem 4.1. Consider a critical decomposable branching process in an IID environment satisfying (2.3) (4.1), (4.2), and

$$
\mathbb{E}\left[\mu_{1}^{-1}\right]<\infty
$$

If for some positive $\alpha$

$$
\begin{aligned}
\mathbb{P}\left[\theta_{1}<\frac{1}{x}\right] & =o\left((\log x)^{-3-\alpha}\right), \quad x \longrightarrow \infty, \\
\mathbb{P}\left[\theta_{1}>x\right] & =o\left((\log x)^{-3-\alpha}\right), \quad x \longrightarrow \infty, \\
\mathbb{P}\left[\theta_{2}>x \theta_{1}\right] & =o\left((\log x)^{-3-\alpha}\right), \quad x \longrightarrow \infty,
\end{aligned}
$$

then there exists a constant $K_{0}$ such that

$$
\mathbb{P}\left[Z_{n}>0\right] \sim \frac{K_{0}}{\log n}, \quad n \longrightarrow \infty .
$$

Before turning to the proof, we make some comments on the conditions and statement of this theorem. 
Notation 4. We will often use the abbreviations $x_{a}:=(\log x)^{2+a}$ and $n_{a}:=(\log n)^{2+a}$.

Condition (4.5) is needed for the following properties to hold for any fixed $\varepsilon>0$; recall notation agreements Notations 2 and 4 ,

$$
\begin{aligned}
\mathbb{P}\left[\min _{0 \leq k \leq x_{\alpha}} \theta_{1, k}<x^{-\varepsilon}\right] & =o\left(\frac{1}{\log x}\right), \quad x \longrightarrow \infty, \\
\mathbb{P}\left[\max _{0 \leq k \leq x_{\alpha}} \theta_{1, k}>x^{\varepsilon}\right] & =o\left(\frac{1}{\log x}\right), \quad x \longrightarrow \infty, \\
\mathbb{P}\left[\max _{0 \leq k \leq x_{\alpha}}\left(\frac{\theta_{2, k}}{\theta_{1, k}}\right)>x^{\varepsilon}\right] & =o\left(\frac{1}{\log x}\right), \quad x \longrightarrow \infty .
\end{aligned}
$$

Each of them is proven via an intermediate step like

$$
\mathbb{P}\left[\min _{0 \leq k \leq x_{\alpha}} \theta_{1, k}<x^{-\varepsilon}\right] \leq x_{\alpha} \mathbb{P}\left[\theta_{1}<x^{-\varepsilon}\right]
$$

relying on the IID assumption for consecutive environmental states. The constant $K_{0}$ in the statement of Theorem 4.1 is the same as in the asymptotic formula from [15] concerning the total number $S_{\mathrm{T}}$ of particles of type 1 ever appeared in the process:

$$
\mathbb{P}\left[S_{\mathrm{T}}>x\right] \sim \frac{K_{0}}{\log x}, \quad x \longrightarrow \infty .
$$

This constant has a complicated nature and is not further explained here. It is necessary to mention that the representation (4.11) has been proved in [15] under conditions (4.1), (4.2), and (4.4) only for the case when the probability generating functions $f_{n}(s, 1)$ are linear fractional with probability 1 . However, the latter restriction is easily removed using the results established later on for the general case in $[12,14]$.

Our proof of Theorem 4.1 uses the next lemma.

Lemma 4.2. Consider conditional moments of the entity $W_{n}$ defined at the beginning of Section 2:

$$
S_{n}^{(i)}:=\sum_{k=0}^{n-1} X_{k} \theta_{i, k}, \quad i=1,2
$$

Under conditions (4.1), (4.5),

$$
\mathbb{P}\left[S_{\mathrm{T}}^{(1)}>x\right] \sim \frac{K_{0}}{\log x}, \quad x \longrightarrow \infty
$$

For any fixed $\epsilon>0$, in Notation 4 ,

$$
\mathbb{P}\left[S_{\mathrm{T}}^{(2)}>n^{\varepsilon} S_{\mathrm{T}}^{(1)} ; T \leq n_{\alpha}\right]=o\left(\frac{1}{\log n}\right), \quad n \longrightarrow \infty
$$


Proof. For any fixed $\epsilon>0$,

$$
\begin{aligned}
\mathbb{P}\left[S_{\mathrm{T}}^{(1)}>x\right] & \geq \mathbb{P}\left[S_{\mathrm{T}}^{(1)}>x ; T \leq x_{\alpha} ; \min _{0 \leq k \leq T} \theta_{1, k}>x^{-\varepsilon}\right] \\
& \geq \mathbb{P}\left[S_{\mathrm{T}}>x^{1+\varepsilon}\right]-\mathbb{P}\left[T>x_{\alpha}\right]-\mathbb{P}\left[\min _{0 \leq k \leq x_{\alpha}} \theta_{1, k} \leq x^{-\varepsilon}\right] .
\end{aligned}
$$

Notice that according to (4.3)

$$
\mathbb{P}\left[T>(\log x)^{2+\varepsilon}\right]=o\left(\frac{1}{\log x}\right), \quad x \longrightarrow \infty, \text { for any fixed } \varepsilon>0
$$

Thus, using (4.7) and (4.11) we get

$$
\liminf _{x \rightarrow \infty}\left\{\log x \cdot \mathbb{P}\left[S_{\mathrm{T}}^{(1)}>x\right]\right\} \geq \liminf _{x \rightarrow \infty}\left\{\log x \cdot \mathbb{P}\left[S_{\mathrm{T}}>x^{1+\varepsilon}\right]\right\} \geq \frac{K_{0}}{(1+\epsilon)}
$$

To obtain a similar estimate from the previously mentioned we write, recalling Notation 4,

$$
\begin{aligned}
\mathbb{P}\left[S_{\mathrm{T}}^{(1)}>x\right] \leq & \mathbb{P}\left[S_{\mathrm{T}}^{(1)}>x ; T \leq x_{\alpha} ; \max _{0 \leq k \leq T} \theta_{1, k} \leq x^{\varepsilon}\right] \\
& +\mathbb{P}\left[T>x_{\alpha}\right]+\mathbb{P}\left[T \leq x_{\alpha} ; \max _{0 \leq k \leq T} \theta_{1, k}>x^{\varepsilon}\right] \\
\leq & \mathbb{P}\left[S_{\mathrm{T}}>x^{1-\varepsilon}\right]+\mathbb{P}\left[T>x_{\alpha}\right]+\mathbb{P}\left[\max _{0 \leq k \leq x_{\alpha}} \theta_{1, k}>x^{\varepsilon}\right],
\end{aligned}
$$

which together with (4.8), (4.11), and (4.16) yields

$$
\begin{aligned}
\limsup _{x \rightarrow \infty}\left\{\log x \cdot \mathbb{P}\left[S_{\mathrm{T}}^{(1)}>x\right]\right\} & \leq \limsup _{x \rightarrow \infty}\left\{\log x \cdot \mathbb{P}\left[S_{\mathrm{T}}>x^{1-\varepsilon}\right]\right\} \\
& \leq \frac{K_{0}}{(1-\epsilon)}
\end{aligned}
$$

Finally, according to (4.9)

$$
\begin{aligned}
\mathbb{P}\left[S_{\mathrm{T}}^{(2)}>n^{\varepsilon} S_{\mathrm{T}}^{(1)} ; T \leq n_{\alpha}\right] & \leq \mathbb{P}\left[\max _{1 \leq k \leq T}\left(\frac{\theta_{2, k}}{\theta_{1, k}}\right)>n^{\varepsilon} ; T \leq n_{\alpha}\right] \\
& =o\left(\frac{1}{\log n}\right), \quad n \longrightarrow \infty .
\end{aligned}
$$


International Journal of Stochastic Analysis

Proof of Theorem 4.1. We will show that

$$
\limsup _{n \rightarrow \infty}\left\{\log n \cdot \mathbb{P}\left[Z_{n}>0\right]\right\} \leq K_{0} \leq \liminf _{n \rightarrow \infty}\left\{\log n \cdot \mathbb{P}\left[Z_{n}>0\right]\right\}
$$

using a counterpart of (2.9)

$$
\begin{aligned}
\mathbb{P}\left[Z_{n}>0\right] & =\mathbb{E}\left[1-\prod_{k=0}^{n-1} Q_{n-k}^{Y_{k}}\right]=\mathbb{E}\left[1-\prod_{k=0}^{n-1} f_{k}^{X_{k}}\left(1, Q_{n-k}\right)\right] \\
& =\mathbb{E}\left[1-\exp \left\{\sum_{k=0}^{n-1} X_{k} \log f_{k}\left(1, Q_{n-k}\right)\right\}\right]
\end{aligned}
$$

and Lemma 4.2.

First we prove the second inequality in (4.21). It follows from (4.22) and the monotonicity of $Q_{n}$ that for any fixed $\epsilon \in(0,1)$

$$
\mathbb{P}\left[Z_{n}>0\right] \geq \mathbb{E}\left[1-\exp \left\{\sum_{k=0}^{T-1} X_{k} \log f_{k}\left(1, Q_{n}\right)\right\} ; S_{\mathrm{T}}^{(2)} \leq n^{\varepsilon} S_{\mathrm{T}}^{(1)}, T \leq n_{\alpha}\right]
$$

Recall that $\log (1-x) \leq-x$ and

$$
f(1, s) \leq 1+\theta_{1}(s-1)+\frac{\theta_{2}}{2}(1-s)^{2}
$$

with the latter inequality being valid thanks to the monotonicity of the second derivative of the generating function. Therefore,

$$
\begin{aligned}
\log f(1, s) & \leq-\theta_{1}(1-s)+\left(\frac{\theta_{2}}{2}\right)(1-s)^{2} \\
\sum_{k=0}^{T-1} X_{k} \log f_{k}\left(1, Q_{n}\right) & \leq-\left(1-Q_{n}\right) \sum_{k=0}^{T-1} X_{k} \theta_{1, k}+\frac{\left(1-Q_{n}\right)^{2}}{2} \sum_{k=0}^{T-1} X_{k} \theta_{2, k} \\
& \leq-c_{1} n^{-1} \sum_{k=0}^{T-1} X_{k} \theta_{1, k}+c_{2} n^{-2} \sum_{k=0}^{T-1} X_{k} \theta_{2, k}
\end{aligned}
$$

where the last inequality is due to (2.5). It follows that given $S_{\mathrm{T}}^{(2)} \leq n^{\varepsilon} S_{\mathrm{T}}^{(1)}$ ，

$$
\sum_{k=0}^{T-1} X_{k} \log f_{k}\left(1, Q_{n}\right) \leq-c n^{-1} S_{\mathrm{T}}^{(1)}
$$


for sufficiently large $n$. As a result, we see that for large $n$

$$
\begin{aligned}
\mathbb{P}\left[Z_{n}>0\right] & \geq \mathbb{E}\left[1-e^{-c n^{-1} S_{\mathrm{T}}^{(1)}} ; S_{\mathrm{T}}^{(2)} \leq n^{\varepsilon} S_{\mathrm{T}}^{(1)}, T \leq n_{\alpha}\right] \\
& \geq \mathbb{E}\left[1-e^{-c n^{-1} S_{\mathrm{T}}^{(1)}}\right]-\mathbb{P}\left[T>n_{\alpha}\right]-\mathbb{P}\left[S_{\mathrm{T}}^{(2)}>n^{\varepsilon} S_{\mathrm{T}}^{(1)} ; T \leq n_{\alpha}\right] .
\end{aligned}
$$

Now, to finish the proof of the second inequality in (4.21) it remains to use (4.16), Lemma 4.2, and

$$
\mathbb{E}\left[1-e^{-\lambda S_{\mathrm{T}}^{(1)}}\right] \sim \frac{K_{0}}{\log (1 / \lambda)}, \quad \lambda \longrightarrow 0,
$$

which again due to Lemma 4.2 follows from the Tauberian theorem [16, Chapter XIII.5, Theorem 4] applied to the right hand side of

$$
\lambda^{-1} \mathbb{E}\left[1-e^{-\lambda S_{\mathrm{T}}^{(1)}}\right]=\int_{0}^{\infty} \mathbb{P}\left[S_{\mathrm{T}}^{(1)}>x\right] e^{-\lambda x} d x
$$

Next, we verify the first inequality in (4.21). From the estimates $\log (1-x) \geq-2 x$, valid for $x \in(0,1 / 2)$, and $f(1, s) \geq 1+\theta_{1}(s-1)$, we conclude that for all sufficiently large $n$

$$
\begin{aligned}
\mathbb{E}[1 & \left.-\exp \left\{\sum_{k=0}^{T-1} X_{k} \log f_{k}\left(1, Q_{n-T}\right)\right\} ; T \leq n_{\alpha} ; \max _{0 \leq k \leq T} \theta_{1, k} \leq n^{\varepsilon}\right] \\
& \leq \mathbb{E}\left[1-\exp \left\{\sum_{k=0}^{T-1} X_{k} \log \left(1-\frac{c \theta_{1, k}}{n}\right)\right\} ; T \leq n_{\alpha} ; \max _{0 \leq k \leq T} \theta_{1, k} \leq n^{\varepsilon}\right] \\
& \leq \mathbb{E}\left[1-\exp \left\{-2 c n^{-1} \sum_{k=0}^{T-1} X_{k} \theta_{1, k}\right\} ; T \leq n_{\alpha} ; \max _{0 \leq k \leq T} \theta_{1, k} \leq n^{\varepsilon}\right] \\
& \leq \mathbb{E}\left[1-e^{-2 c n^{\varepsilon-1} S_{\mathrm{T}}^{(1)}}\right] .
\end{aligned}
$$

Thus,

$$
\begin{aligned}
\mathbb{P}\left[Z_{n}>0\right] & \leq \mathbb{E}\left[1-\exp \left\{\sum_{k=0}^{T-1} X_{k} \log f_{k}\left(1, Q_{n-T}\right)\right\}\right] \\
& \leq \mathbb{E}\left[1-e^{-2 c n^{\varepsilon-1} S_{\mathrm{T}}^{(1)}}\right]+\mathbb{P}\left[T>n_{\alpha}\right]+\mathbb{P}\left[\max _{0 \leq k \leq n_{\alpha}} \theta_{1, k}>n^{\varepsilon}\right],
\end{aligned}
$$

and (4.21) follows due to (4.8) and (4.16). 
International Journal of Stochastic Analysis

\section{The Subcritical Case with an IID Environment}

We continue studying BGW processes in IID environment, but now assume that

$$
\mathbb{E}[\zeta]<0, \quad \operatorname{Var}[\zeta] \in(0, \infty)
$$

instead of (4.1). Results rely upon a theorem from [17] giving the asymptotics for $\mathbb{P}\left[W_{\mathrm{T}}>x\right]$ as $x \rightarrow \infty$. It requires an important technical assumption, namely, the existence of a constant $\kappa$ such that

$$
\mathbb{E}\left[e^{\kappa \zeta}\right]=\mathbb{E}\left[\mu_{1}^{\kappa}\right]=1, \quad 0<\kappa<\infty
$$

If, in addition, for some $\delta>0$

$$
0<\mathbb{E}\left[\xi_{2}^{\kappa+\delta}\right]<\infty, \quad \mathbb{E}\left[\theta_{1}^{\kappa}\right]<\infty,
$$

and either

$$
\kappa>1, \quad \mathbb{E}\left[\left|\xi_{1}\right|^{\kappa}\right]<\infty,
$$

or

$$
0<\kappa \leq 1, \quad \mathbb{E}\left[\left|\mu_{2}-\mu_{1}^{2}\right|^{\kappa}+\left|\theta_{2}-\theta_{1}^{2}\right|^{\kappa}\right]<\infty,
$$

then, according to [17], there exists a constant $C_{\kappa} \in(0, \infty)$ such that

$$
\mathbb{P}\left[W_{\mathrm{T}}>x\right] \sim C_{\kappa} x^{-\kappa}, \quad x \longrightarrow \infty
$$

It is also known [18-20] that under (5.1) and (5.2)

$$
\mathbb{P}\left[X_{n}>0\right]=\mathbb{P}[T>n]=o\left(A^{n}\right) \text { for some constant } A \in(0,1) .
$$

Theorem 5.1. If conditions (5.1), (5.2), (5.3), and either (5.4) or (5.5) hold, then

$$
\mathbb{P}\left[Z_{n}>0\right] \sim K_{\kappa} \cdot q_{\kappa}(n), \quad n \longrightarrow \infty,
$$

for some positive constant $K_{\kappa}$, given by (5.15) latter, where

$$
q_{\kappa}(n)= \begin{cases}n^{-\kappa} & \text { if } \kappa<1 \\ n^{-1} \log n & \text { if } \kappa=1 \\ n^{-1} & \text { if } \kappa>1 .\end{cases}
$$


Proof. Referring to (5.7), put $B_{n}:=2 \log n / \log \left(A^{-1}\right)$ and notice that

$$
\mathbb{P}\left[T>B_{n}\right]=o\left(n^{-2}\right), \quad n \longrightarrow \infty
$$

From the first equality in (4.22) and the evident inequality $Q_{n-k} \leq Q_{n}$ we obtain for $n \geq B_{n}$

$$
\begin{aligned}
\mathbb{P}\left[Z_{n}>0\right] & \geq \mathbb{E}\left[1-\prod_{k=0}^{n-1} Q_{n}^{Y_{k}} ; T \leq B_{n}\right] \\
& =\mathbb{E}\left[1-e^{W_{\mathrm{T}} \log Q_{n}} ; T \leq B_{n}\right] \geq \mathbb{E}\left[1-e^{W_{\mathrm{T}} \log Q_{n}}\right]-\mathbb{P}\left[T>B_{n}\right] .
\end{aligned}
$$

On the other hand, we have a similar upper bound

$$
\begin{aligned}
\mathbb{P}\left[Z_{n}>0\right] & \leq \mathbb{E}\left[1-Q_{n-T}^{W_{\mathrm{T}}} ; T \leq B_{n}\right]+\mathbb{P}\left[T>B_{n}\right] \\
& \leq \mathbb{E}\left[1-e^{W_{\mathrm{T}} \log Q_{n-N \log n}}\right]+\mathbb{P}\left[T>B_{n}\right] .
\end{aligned}
$$

It remains to observe that due to (2.5) and (5.6), the same Tauberian theorem [16, Chapter XIII.5, Theorem 4] applied to the right-hand side of

$$
\lambda^{-1} \mathbb{E}\left[1-e^{-\lambda W_{\mathrm{T}}}\right]=\int_{0}^{\infty} \mathbb{P}\left[W_{\mathrm{T}}>x\right] e^{-\lambda x} d x
$$

yields

$$
\mathbb{E}\left[1-e^{W_{\mathrm{T}} \log Q_{n}}\right] \sim K_{\kappa} \cdot q_{\kappa}(n), \quad n \longrightarrow \infty,
$$

with

$$
K_{\kappa}= \begin{cases}\Gamma(1-\kappa) C_{\kappa}\left(\frac{2}{m_{2}}\right)^{\kappa} & \text { if } \kappa<1 \\ \frac{2}{m_{2}} C_{1} & \text { if } \kappa=1 \\ \frac{2}{m_{2}} \int_{0}^{\infty} \mathbb{P}\left(W_{\mathrm{T}}>x\right) d x & \text { if } \kappa>1\end{cases}
$$

\section{The Critical Case with a Markovian Environment}

As compared to the IID case, Markovian environments require extra conditions on the underlying Markov chain. First we assume that the two-type critical process $\left(X_{n}, Z_{n}\right)$ evolves 
in a stationary Markovian random environment as defined in Section 3.2. Besides, we suppose the validity of (3.11) and that for some $\rho>0$

$$
\mathbb{P}[\tau>x]=o\left(x^{-1}(\log x)^{-1-\rho}\right), \quad x \longrightarrow \infty
$$

This implies that $a:=\mathbb{E}[\tau]<\infty$ and due to Lemma 3.1 conditions $\mathbb{E}[\widehat{\zeta}]=0$ and $\mathbb{E}[\zeta]=0$ become equivalent. Moreover, under condition (6.1) the sequence of regeneration times (3.4) satisfies

$$
\mathbb{P}\left[\left|k^{-1} \tau_{k}-a\right|>\varepsilon\right]=o\left((\log k)^{-1-\rho}\right), \quad k \longrightarrow \infty,
$$

for an arbitrarily small $\varepsilon>0$, cf. [21].

Theorem 6.1. Assume (2.3), (3.11), (6.1), and

$$
\begin{gathered}
\mathbb{E}[\zeta]=0, \quad \operatorname{Var}[\hat{\zeta}] \in(0, \infty), \\
\mathbb{E}\left[\widehat{\mu}_{2} \widehat{\mu}_{1}^{-2}\left(1+\max \left(0, \log \widehat{\mu}_{1}\right)\right)\right]<\infty, \quad \mathbb{E}\left[\widehat{\mu}_{1}^{-1}\right]<\infty .
\end{gathered}
$$

Further, for some positive a let

$$
\begin{aligned}
& \mathbb{P}\left[\widehat{\theta}_{1}<\frac{1}{x}\right]=o\left((\log x)^{-3-\alpha}\right), \quad x \longrightarrow \infty, \\
& \mathbb{P}\left[\widehat{\theta}_{1}>x\right]=o\left((\log x)^{-3-\alpha}\right), \quad x \longrightarrow \infty, \\
& \mathbb{P}\left[\widehat{\theta}_{2}>x \widehat{\theta}_{1}\right]=o\left((\log x)^{-3-\alpha}\right), \quad x \longrightarrow \infty \text {. }
\end{aligned}
$$

Then $\mathbb{P}\left[X_{n}>0\right]=O\left(n^{-1 / 2}\right)$, and there exists a constant $\widehat{K}_{0}>0$ such that

$$
\mathbb{P}\left[Z_{n}>0\right] \sim \frac{\widehat{K}_{0}}{\log n}, \quad n \longrightarrow \infty .
$$

Proof. The statement is divided into two steps: first

$$
\mathbb{P}\left[\widehat{Z}_{r}>0\right] \sim \frac{\widehat{K}_{0}}{\log r}, \quad r \longrightarrow \infty
$$

and then

$$
\mathbb{P}\left[X_{n}+Z_{n}>0\right] \sim \frac{\widehat{K}_{0}}{\log n}, \quad n \longrightarrow \infty,
$$


together with

$$
\mathbb{P}\left[X_{n}>0\right]=O\left(n^{-1 / 2}\right), \quad n \longrightarrow \infty
$$

Fix $\delta \in(0,1 / 4)$, and write

$$
\begin{aligned}
\mathbb{P}\left[\widehat{Z}_{r}>0\right]= & \mathbb{P}\left[\widehat{Z}_{r}>0 ; \tau_{r^{\delta}} \leq r^{2 \delta},\left|\tau_{r}-r a\right| \leq r^{1-\delta}\right] \\
& +O\left(\mathbb{P}\left[\tau_{r^{\delta}}>r^{2 \delta}\right]\right)+O\left(\mathbb{P}\left[\left|r^{-1} \tau_{r}-a\right|>r^{-\delta}\right]\right) .
\end{aligned}
$$

Here the last two terms are treated with the help of $\mathbb{P}\left(\tau_{r^{6}}>r^{2 \delta}\right) \leq a r^{-\delta}$ and (6.2), while the main term is analyzed by means of ideas from the proof of Theorem 4.1. Letting $\widehat{Y}_{k}$ be the number of type $\widehat{2}$ daughters produced by $\widehat{X}_{k}$ particles of type $\hat{1}$ and putting $\widehat{T}:=\min \left\{r: \widehat{Z}_{r}=0\right\}$, we deduce from

$$
\mathbb{P}\left[\widehat{Z}_{r}>0 ; \tau_{r^{\delta}} \leq r^{2 \delta},\left|\tau_{r}-r a\right| \leq r^{1-\delta}\right]=\mathbb{E}\left[1-\prod_{k=0}^{r-1} Q_{\tau_{r}-\tau_{k}}^{\widehat{Y}_{k}} ; \tau_{r^{\delta}} \leq r^{2 \delta},\left|\tau_{r}-r a\right| \leq r^{1-\delta}\right]
$$

a lower bound

$$
\begin{aligned}
\mathbb{P}\left[\widehat{Z}_{r}>0 ; \tau_{r^{\delta}} \leq r^{2 \delta},\left|\tau_{r}-r a\right| \leq r^{1-\delta}\right] \\
\geq \mathbb{E}\left[1-\prod_{k=0}^{\widehat{T}-1} Q_{\tau_{r}-\tau_{k}}^{\hat{Y}_{k}}, \widehat{T} \leq r^{\delta}, \tau_{r^{\delta}} \leq r^{2 \delta},\left|\tau_{r}-r a\right| \leq r^{1-\delta}\right] \\
\geq \mathbb{E}\left[1-\prod_{k=0}^{\widehat{T}-1} f_{k}^{\hat{X}_{k}}\left(1, Q_{2 r a}\right) ; \widehat{T} \leq r^{\delta}, \tau_{r^{\delta}} \leq r^{2 \delta},\left|r^{-1} \tau_{r}-a\right| \leq r^{-\delta}\right] \\
\geq \mathbb{E}\left[1-\prod_{k=0}^{\widehat{T}-1} f_{k}^{\hat{X}_{k}}\left(1, Q_{2 r a}\right)\right]-\mathbb{P}\left(\widehat{T}>r^{\delta}\right)-\mathbf{P}\left(\tau_{r^{\delta}}>r^{2 \delta}\right)-\mathbb{P}\left(\left|r^{-1} \tau_{r}-a\right|>r^{-\delta}\right) \\
=\mathbb{E}\left[1-\prod_{k=0}^{\widehat{T}-1} f_{k}^{\widehat{X}_{k}}\left(1, Q_{2 r a}\right)\right]+o\left(\frac{1}{\log ^{1+\rho} r}\right) .
\end{aligned}
$$

Hence, applying arguments used to derive (4.21) in Theorem 4.1 one can show that for some $\widehat{K}_{0}>0$

$$
\limsup _{r \rightarrow \infty}\left\{\log r \cdot \mathbb{P}\left[\widehat{Z}_{r}>0\right]\right\} \leq \widehat{K}_{0} \leq \liminf _{r \rightarrow \infty}\left\{\log r \cdot \mathbb{P}\left[\widehat{Z}_{r}>0\right]\right\}
$$

proving (6.6). 
International Journal of Stochastic Analysis

To demonstrate that (6.7) follows from (6.6) observe first that due to

$$
\mathbb{P}\left[\widehat{X}_{r}>0\right]=O\left(r^{-1 / 2}\right), \quad r \longrightarrow \infty
$$

we have

$$
\mathbb{P}\left[\widehat{X}_{r}+\widehat{Z}_{r}>0\right] \sim \frac{\widehat{K}_{0}}{\log r}, \quad r \longrightarrow \infty
$$

Setting $N_{n}:=\max \left\{k: \tau_{k} \leq n\right\}$ we obtain

$$
\mathbb{P}\left[\widehat{X}_{N_{n}+1}+\widehat{Z}_{N_{n}+1}>0\right] \leq \mathbb{P}\left[X_{n}+Z_{n}>0\right] \leq \mathbb{P}\left[\widehat{X}_{N_{n}}+\widehat{Z}_{N_{n}}>0\right]
$$

and for any $\varepsilon \in(0,1)$ we get

$$
\begin{aligned}
\mathbb{P}\left[\widehat{X}_{N_{n}}+\widehat{Z}_{N_{n}}>0\right]= & \mathbb{P}\left[\widehat{X}_{N_{n}}+\widehat{Z}_{N_{n}}>0 ; N_{n} \geq a^{-1} n(1-\varepsilon)\right] \\
& +O\left(\mathbb{P}\left[N_{n}<a^{-1} n(1-\varepsilon)\right]\right) .
\end{aligned}
$$

It follows that

$$
\mathbb{P}\left[\widehat{X}_{N_{n}}+\widehat{Z}_{N_{n}}>0 ; N_{n} \geq a^{-1} n(1-\varepsilon)\right] \leq \mathbb{P}\left[\widehat{X}_{a^{-1} n(1-\varepsilon)}+\widehat{Z}_{a^{-1} n(1-\varepsilon)}>0\right] .
$$

On the other hand, again by (6.2) as $n \rightarrow \infty$

$$
\mathbb{P}\left[N_{n}<a^{-1} n(1-\varepsilon)\right]=\mathbb{P}\left[\tau_{a^{-1} n(1-\varepsilon)}>n\right]=o\left((\log n)^{-1-\rho}\right)
$$

Thus,

$$
\limsup _{n \rightarrow \infty}\left\{\log n \cdot \mathbb{P}\left[X_{n}+Z_{n}>0\right]\right\} \leq \limsup _{n \rightarrow \infty}\left\{\log n \cdot \mathbb{P}\left[\widehat{X}_{a^{-1} n(1-\varepsilon)}+\widehat{Z}_{a^{-1} n(1-\varepsilon)}>0\right]\right\} \leq \widehat{K}_{0}
$$

A similar estimate shown latter follows from

$$
\begin{aligned}
\mathbb{P}\left[\widehat{X}_{N_{n}+1}+\widehat{Z}_{N_{n}+1}>0\right] & \geq \mathbb{P}\left[\widehat{X}_{a^{-1} n(1+\varepsilon)}+\widehat{Z}_{a^{-1} n(1+\varepsilon)}>0 ; N_{n}+1 \leq a^{-1} n(1+\varepsilon)\right] \\
& =\mathbb{P}\left[\widehat{X}_{a^{-1} n(1+\varepsilon)}+\widehat{Z}_{a^{-1} n(1+\varepsilon)}>0\right]+o\left((\log n)^{-1-\rho}\right) .
\end{aligned}
$$

Finally, relation (6.8) is derived from (6.13) by the law of large numbers argument. 


\section{Subcritical Processes with a Markovian Environment}

Assume now that the two-type subcritical process $\left(X_{n}, Z_{n}\right)$ evolves in a stationary Markovian random environment as defined in Section 3.2. Here, similarly to Section 6 the auxiliary branching process $\left(\widehat{X}_{r}, \widehat{Z}_{r}\right)$ in IID environment with probability generating functions (3.6) and (3.7) plays an important role.

Single-type subcritical processes with a Markovian environment were recently studied in [22]. According to [22] under the conditions of our next theorem one has, similarly to (5.7), that

$$
\mathbb{P}\left[X_{n}>0\right]=o\left(A^{n}\right) \quad \text { for some constant } A \in(0,1)
$$

Theorem 7.1. Assume that assumption (2.3) holds,

$$
\mathbb{E}[\zeta]<0, \quad \operatorname{Var}[\hat{\zeta}] \in(0, \infty),
$$

and conditions (5.2), (5.3) and either (5.4) or (5.5) are valid for the corresponding random variables related to the embedded process $\left(\widehat{X}_{r}, \widehat{Z}_{r}\right)$ with the key constant $\kappa$ replaced by $\widehat{\mathcal{\kappa}}>0$. Suppose, in addition, that

$$
\mathbb{P}[\tau>x]=o\left(x^{-1-\min (\hat{\kappa}, 1)}\right), \quad x \longrightarrow \infty
$$

Then, there exists a constant $\widehat{K} \equiv \widehat{K}_{\widehat{\kappa}}>0$, given by (7.16) and (7.20) latter, such that, see (5.9),

$$
\mathbb{P}\left[Z_{n}>0\right] \sim a^{\min (1, \widehat{\kappa})} \widehat{K} q_{\hat{\kappa}}(n), \quad n \longrightarrow \infty
$$

Proof. Our main arguments here are similar to that used in the proof of Theorem 6.1. Fix $\varepsilon \in(0,1)$ and a sufficiently large $N$ and with $a=\mathbb{E}(\tau)$ write

$$
\mathbb{P}\left[\widehat{Z}_{r}>0\right]=\mathbb{P}\left[\hat{Z}_{r}>0 ; \mathbb{B}(r, \varepsilon)\right]+O\left(\mathbb{P}\left[\tau_{N \log r}>r^{\widehat{\kappa}} \log ^{3} r\right]+\mathbb{P}\left[\left|\tau_{r}-r a\right|>r \varepsilon\right]\right),
$$

where

$$
\mathcal{B}(r, \varepsilon):=\left\{\tau_{N \log r} \leq r^{\widehat{\kappa}} \log ^{3} r,\left|\tau_{r}-r a\right| \leq \varepsilon r\right\}
$$

Clearly,

$$
\mathbb{P}\left[\tau_{N \log r}>r^{\widehat{\kappa}} \log ^{3} r\right] \leq \frac{N a}{r^{\widehat{\kappa}} \log ^{2} r}=o\left(r^{-\widehat{\kappa}}\right)
$$

Further, if $\widehat{\kappa}<1$ then according to [21] under condition (7.3) we have

$$
\mathbb{P}\left[\left|\tau_{r}-r a\right|>\varepsilon r\right]=o\left(r^{-\widehat{\kappa}}\right)
$$


Thus,

$$
\mathbb{P}\left[\widehat{Z}_{r}>0\right] \geq \mathbb{P}\left[\widehat{Z}_{r}>0 ; \Re(r, \varepsilon)\right]=\mathbb{E}\left[1-\prod_{k=0}^{r-1} Q_{\tau_{r}-\tau_{k}}^{\widehat{Y}_{k}} ; ß(r, \varepsilon)\right]
$$

and therefore, denoting by $\bar{B}(r, \varepsilon)$ the event complementary to $\mathcal{B}(r, \varepsilon)$, we get

$$
\begin{aligned}
& \mathbb{P}\left[\widehat{Z}_{r}>0\right] \geq \mathbb{E}\left[1-\prod_{k=0}^{\widehat{T}-1} Q_{\tau_{r}-\tau_{k}}^{\widehat{Y}_{k}} ; \widehat{T} \leq N \log r ; ß(r, \varepsilon)\right] \\
& \geq \mathbb{E}\left[1-\prod_{k=0}^{\widehat{T}-1} Q_{r a+2 \varepsilon r}^{\widehat{Q}_{k}} ; \widehat{T} \leq N \log r ; ß(r, \varepsilon)\right] \\
& =\mathbb{E}\left[1-e^{\widehat{W}_{\mathrm{T}} \log Q_{r a+2 \varepsilon r}}\right]-\mathbb{P}[\widehat{T}>N \log r]-\mathbb{P}[\overline{\mathcal{B}}(r, \varepsilon)] \text {. }
\end{aligned}
$$

Due to (1.2) and (7.2)

$$
\mathbb{P}\left[\widehat{X}_{r}>0\right]=\mathbb{P}[\widehat{T}>r]=o\left(A^{r}\right) \quad \text { for some } A<1
$$

It follows that in view of (1.2), (7.7), and (7.8) one can find $N$ such that

$$
\mathbb{P}[\widehat{T}>N \log r]+\mathbb{P}[\overline{\mathcal{B}}(r, \varepsilon)]=o\left(r^{-\widehat{\kappa}}\right) .
$$

On the other hand, using (2.5) and

$$
\mathbb{P}\left[\widehat{W}_{\mathrm{T}}>y\right] \sim \widehat{C} y^{-\widehat{\kappa}}, \quad \widehat{C} \in(0, \infty),
$$

one can show, arguing as in Theorem 5.1, that for $\widehat{\kappa}<1$

$$
\begin{aligned}
\liminf _{\varepsilon \downarrow 0} \lim _{r \rightarrow \infty} r^{\widehat{\kappa}} \mathbb{E}\left[1-e^{\widehat{W}_{\mathrm{T}} \log Q_{r a+2 r r}}\right] \\
\quad=\liminf _{\varepsilon \downarrow 0} \lim _{r \rightarrow \infty} \frac{r^{\widehat{\kappa}}}{-\log Q_{r a+2 \varepsilon r}} \int_{0}^{\infty} \mathbb{P}\left[\widehat{W}_{\mathrm{T}}>x\right] e^{x \log Q_{r a+2 \varepsilon r}} d x \\
=\hat{C} \Gamma(1-\widehat{\kappa}) \liminf _{\varepsilon \downarrow 0}\left(\frac{2}{m_{2} a(1+2 \varepsilon)}\right)^{\widehat{\kappa}}
\end{aligned}
$$

giving

$$
\liminf _{r \rightarrow \infty} r^{\widehat{\kappa}} \mathbb{P}\left[\widehat{Z}_{r}>0\right] \geq \widehat{K}
$$


with

$$
\widehat{K}=\widehat{C} \Gamma(1-\widehat{\kappa})\left(\frac{2}{m_{2} a}\right)^{\widehat{\kappa}} \text { for } \widehat{\kappa}<1 .
$$

A similar upper bound in view of (1.2) and (7.11) yields

$$
\lim _{r \rightarrow \infty} r^{\widehat{\kappa}} \mathbb{P}\left[\widehat{X}_{r}+\widehat{Z}_{r}>0\right]=\widehat{K}
$$

If $\widehat{\kappa} \geq 1$ then condition (7.3) entails

$$
\mathbb{P}\left[\left|\tau_{r}-r a\right|>\varepsilon r\right]=o\left(r^{-1}\right),
$$

and, as before, this implies

$$
\lim _{r \rightarrow \infty}\left(q_{\widehat{\kappa}}(r)\right)^{-1} \mathbf{P}\left[\widehat{X}_{r}+\widehat{Z}_{r}>0\right]=\widehat{K}
$$

where

$$
\widehat{K}=\frac{2}{m_{2} a} \cdot \begin{cases}\widehat{C}, & \text { if } \widehat{\kappa}=1, \\ \int_{0}^{\infty} \mathbb{P}\left[\widehat{W}_{\mathrm{T}}>x\right] d x, & \text { if } \widehat{\kappa}>1 .\end{cases}
$$

We proceed by recalling (6.15). For any $\varepsilon \in(0,1)$

$$
\begin{aligned}
\mathbb{P}\left[\widehat{X}_{N_{n}}+\widehat{Z}_{N_{n}}>0\right]= & \mathbb{P}\left[\widehat{X}_{N_{n}}+\widehat{Z}_{N_{n}}>0 ; N_{n} \geq a^{-1} n(1-\varepsilon)\right] \\
& +O\left(\mathbb{P}\left[N_{n}<a^{-1} n(1-\varepsilon)\right]\right)
\end{aligned}
$$

and as $n \rightarrow \infty$

$$
\begin{aligned}
& \mathbb{P}\left[\widehat{X}_{N_{n}}+\widehat{Z}_{N_{n}}>0 ; N_{n} \geq a^{-1} n(1-\varepsilon)\right] \\
& \quad \leq \mathbb{P}\left[\widehat{X}_{a^{-1} n(1-\varepsilon)}+\widehat{Z}_{a^{-1} n(1-\varepsilon)}>0\right] \sim q_{\widehat{\kappa}}\left(a^{-1} n(1-\varepsilon)\right) \widehat{K}
\end{aligned}
$$

It follows from [21] and our conditions that

$$
\mathbb{P}\left[N_{n}<a^{-1} n(1-\varepsilon)\right]=\mathbb{P}\left[S_{a^{-1} n(1-\varepsilon)}>n\right]=o\left(n^{-\min (\hat{\kappa}, 1)}\right), \quad n \longrightarrow \infty .
$$

Thus,

$$
\limsup _{n \rightarrow \infty}\left(q_{\widehat{\kappa}}(n)\right)^{-1} \mathbb{P}\left[X_{n}+Z_{n}>0\right] \leq \lim _{\varepsilon \downarrow 0} \limsup _{n \rightarrow \infty}\left(q_{\widehat{\kappa}}(n)\right)^{-1} \mathbb{P}\left[\widehat{X}_{N_{n}}+\widehat{Z}_{N_{n}}>0\right],
$$


so that

$$
\limsup _{n \rightarrow \infty}\left(q_{\widehat{\kappa}}(n)\right)^{-1} \mathbb{P}\left[X_{n}+Z_{n}>0\right] \leq a^{\min (1, \widehat{\kappa})} \widehat{K} .
$$

The corresponding lower bound is obtained similarly.

\section{Acknowledgments}

E. Dyakonova was supported in part by the Program of RAS “Theoretical Problems of Contemporary Mathematics" and by INTAS Grant no. 03-51-5018. S. Sagitov was supported by Swedish Research Council Grant no. 621-2010-5623. V. Vatutin was supported in part by the Program of RAS “Theoretical Problems of Contemporary Mathematics" and by a WennerGren Foundation Visiting Researcher Scholarship.

\section{References}

[1] M. Benaim and S. J. Schreiber, "Persistence of structured population in random environments," Theoretical Population Biology, vol. 76, no. 1, pp. 19-34, 2009.

[2] P. Haccou and Y. Iwasa, "Optimal mixed strategies in stochastic environments," Theoretical Population Biology, vol. 47, no. 2, pp. 212-243, 1995.

[3] S. J. Schreiber, M. Benaïm, and K. A. S. Atchade, "Persistence in fluctuating environments," Journal of Mathematical Biology, vol. 62, no. 5, pp. 655-683, 2011.

[4] S. Sagitov and M. C. Serra, "Multitype Bienaymé-Galton-Watson processes escaping extinction," Advances in Applied Probability, vol. 41, no. 1, pp. 225-246, 2009.

[5] C. Tomasetti, "On the probability of random genetic mutations for various types of tumor growth," Bulletin of Mathematical Biology, vol. 74, no. 6, pp. 1379-1395, 2012.

[6] G. Alsmeyer and S. Grottrup, "A host-parasite model for a two-type cell population," Advances in Applied Probability, vol. 45, 2013.

[7] K. B. Athreya and P. E. Ney, Branching Processes, vol. 196 of Die Grundlehren der mathematischen Wissenschaften, Springer, New York, NY, USA, 1972.

[8] J. Foster and P. Ney, "Decomposable critical multi-type branching processes," Sankhyā. The Indian Journal of Statistics A, vol. 38, no. 1, pp. 28-37, 1976.

[9] V. A. Vatutin and S. M. Sagitov, "A decomposable critical branching process with two types of particles. Probabilistic problems of discrete mathematics," Proceedings of the Steklov Institute of Mathematics, vol. 4, pp. 1-19, 1988.

[10] A. M. Zubkov, "The limit behavior of decomposable critical branching processes with two types of particles," Theory of Probability \& Its Applications, vol. 27, no. 2, pp. 235-237, 1983.

[11] Y. Ogura, "Asymptotic behavior of multitype Galton-Watson processes," Journal of Mathematics of Kyoto University, vol. 15, no. 2, pp. 251-302, 1975.

[12] V. I. Afanasyev, J. Geiger, G. Kersting, and V. A. Vatutin, "Criticality for branching processes in random environment," The Annals of Probability, vol. 33, no. 2, pp. 645-673, 2005.

[13] R. Durrett, Probability: Theory and Examples, Cambridge Series in Statistical and Probabilistic Mathematics, Cambridge University Press, Cambridge, UK, 4th edition, 2010.

[14] J. Geiger and G. Kersting, "The survival probability of a critical branching process in random environment," Theory of Probability \& Its Applications, vol. 45, no. 3, pp. 607-615, 2000.

[15] V. I. Afanasyev, "On the maximum of a critical branching process in a random environment," Discrete Mathematics and Applications, vol. 9, pp. 267-284, 1999.

[16] W. Feller, An Introduction to Probability Theory and Its Applications. II, John Wiley \& Sons, New York, NY, USA, 1966.

[17] V. A. Vatutin, "Polling systems and multitype branching processes in a random environment with a final product," Theory of Probability E Its Applications, vol. 55, no. 4, pp. 631-660, 2011. 
[18] V. I. Afanasyev, J. Geiger, G. Kersting, and V. A. Vatutin, "Functional limit theorems for strongly subcritical branching processes in random environment," Stochastic Processes and Their Applications, vol. 115, no. 10, pp. 1658-1676, 2005.

[19] V. I. Afanasyev, C. Böinghoff, G. Kersting, and V. A. Vatutin, "Limit theorems for weakly subcritical branching processes in random environment," Journal of Theoretical Probability, vol. 25, no. 3, pp. 703732, 2012.

[20] V. I. Afanasyev, C. Boeinghoff, G. Kersting, and V. A. Vatutin, "Conditional limit theorems for intermediately subcritical branching processes in random environment," Annales de l'Institut Henri Poincare (B) Probability and Statistics, In press, http:/ / arxiv.org/abs/1108.2127.

[21] C. C. Heyde and V. K. Rohatgi, "A pair of complementary theorems on convergence rates in the law of large numbers," Proceedings of the Cambridge Philosophical Society, vol. 63, pp. 73-82, 1967.

[22] E. E. Dyakonova, "Multitype Galton-Watson branching processes in Markovian random environment," Theory of Probability and Its Applications, vol. 56, pp. 508-517, 2011. 


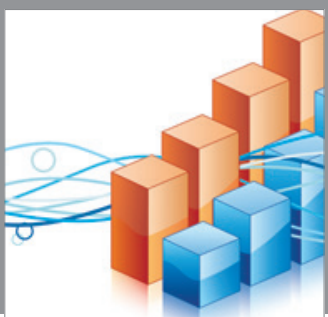

Advances in

Operations Research

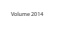

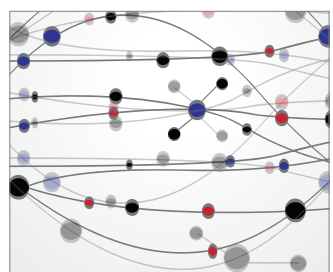

\section{The Scientific} World Journal
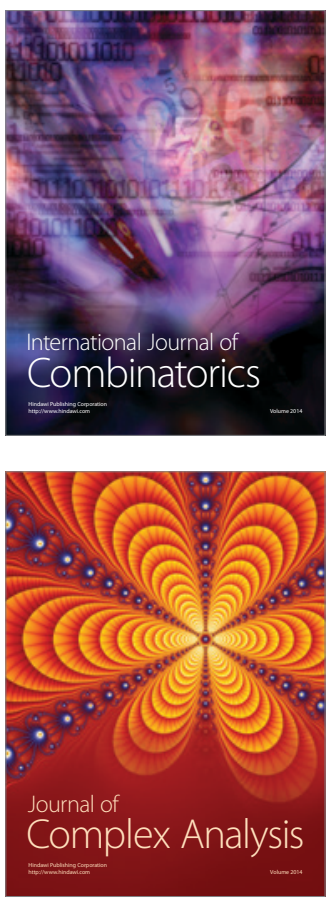

International Journal of

Mathematics and

Mathematical

Sciences
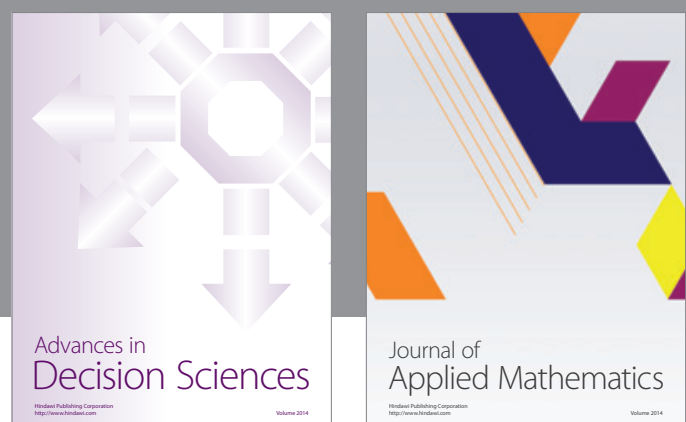

Journal of

Applied Mathematics
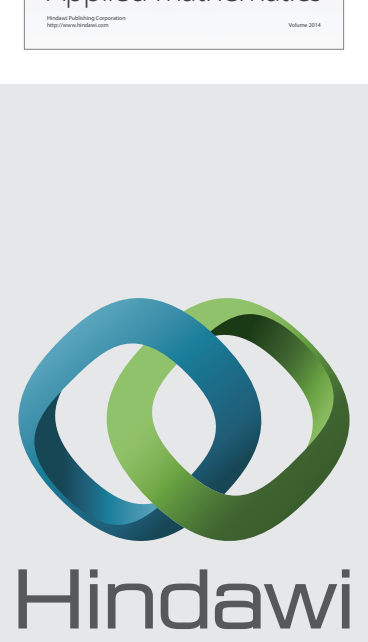

Submit your manuscripts at http://www.hindawi.com
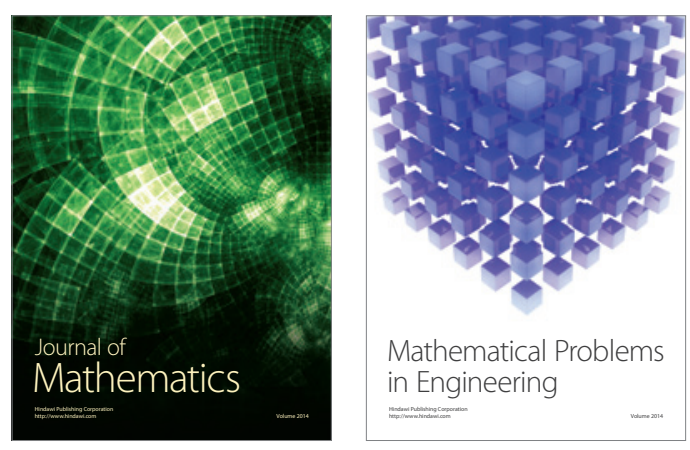

Mathematical Problems in Engineering
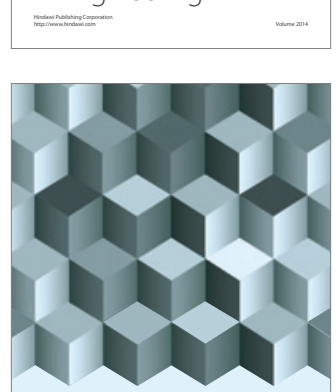

Journal of

Function Spaces
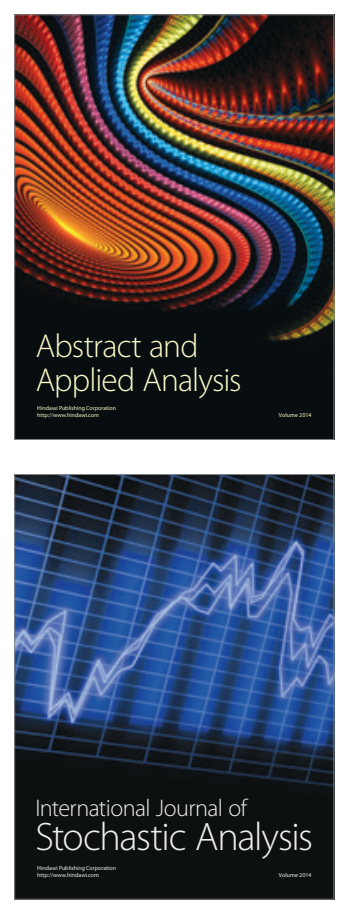

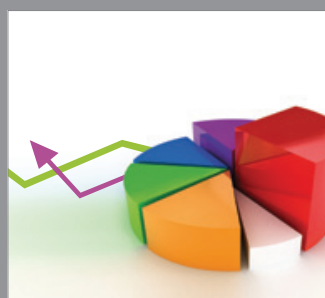

ournal of

Probability and Statistics

Promensencen
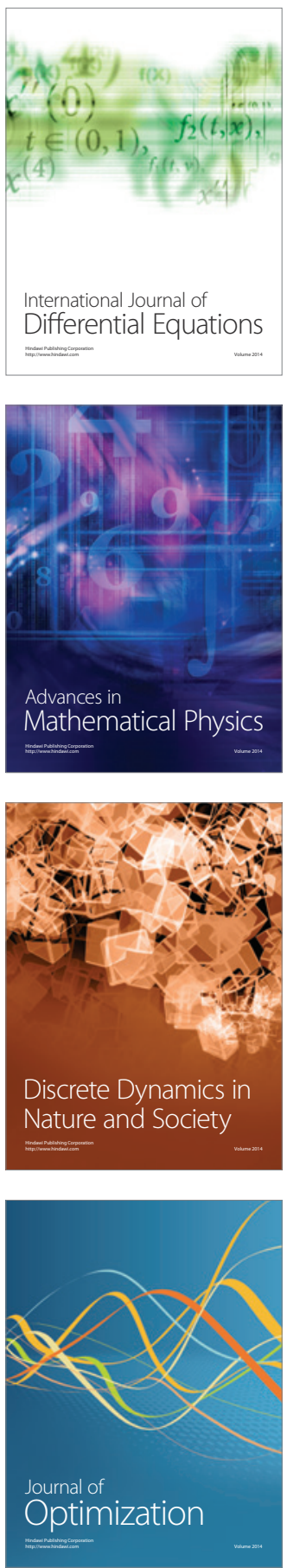